\title{
Electrochemical Properties of Alkanethiol Monolayers Adsorbed on Nanoporous Au Surfaces ${ }^{\dagger}$
}

\author{
Yeonyi Chu, Bora Seo, and Jongwon Kim* \\ Department of Chemistry, Chungbuk National University, Cheongju, Chungbuk 361-763, Korea \\ ${ }^{*}$ E-mail: jongwonkim@chungbuk.ac.kr \\ Received July 19, 2010, Accepted September 15, 2010
}

Key Words: Alkanethiol, Monolayer, Nanoporous Au, Reductive desorption, Electron transfer

Self-assembled monolayers (SAMs) of thiolate molecules on Au surfaces have been the subject of intensive research for the last few decades due to their unique physical and chemical properties. ${ }^{1}$ The well-organized surface structures of thiolate SAMs with various end-group functionalities can be further utilized for many applications in biology and nanotechnology. ${ }^{2}$ In addition to the practical applications, SAMs of thiolate molecules on Au surfaces also provide unique opportunities to address fundamental issues in surface chemistry such as self-organized surface structures, electron transfer behaviors, and moleculesubstrate interactions. ${ }^{3-5}$ Although there have been numerous reports on the fundamental physical and chemical properties of thiolate SAMs on Au surfaces, most of them were investigated on flat Au surfaces, typically on well-defined $\mathrm{Au}(111)$ surfaces. These previous investigations provide valuable insights on the surface phenomena of thiolate adsorption on Au surfaces; however, the effect of the structure of Au substrates on the basic properties of thiolate monolayers such as electrochemical properties and structures of the adsorbed layers has yet to be understood. A few reports have addressed the structures of thiolate SAMs on different single crystalline Au surfaces. ${ }^{3,5,6}$ Porter et al. examined the different binding sites of thiolates on various Au surfaces from voltammetric data for the reductive desorption of alkanethiols.,

Since the stabilization of $\mathrm{Au}$ nanoparticles (AuNPs) with alkanethiol monolayers is one of the most important applications of the thiolate adsorption on Au surfaces, it would be highly desirable to understand the detailed nature of the thiolate monolayers on the surface of AuNPs. As schematically shown in Figure 1A, the intrinsic properties of thiolate monolayers adsorbed on AuNPs would be quite different from those on flat $\mathrm{Au}$ surfaces. The adsorption of thiolate molecules on a flat $\mathrm{Au}$ surface results in the well-organized self-assembled structures through hydrophobic interactions between alkyl chains of adjacent thiolates, while the self-organization is apparently limited on curved AuNP surfaces. However, little attention has been paid to the intrinsic properties of thiolate monolayers on AuNPs, particularly to the electrochemical ones. Here, we report the electrochemical properties of alkanethiol monolayers adsorbed on nanoporous Au (NPG) surfaces. NPG surfaces with welldefined nanostructures consisting of ligaments and pores have attracted much attention due to their applications in hetero-

\footnotetext{
${ }^{\dagger}$ This paper is dedicated to Professor Hasuck Kim for his outstanding contribution to electrochemistry and analytical chemistry.
}

(A)

(B)
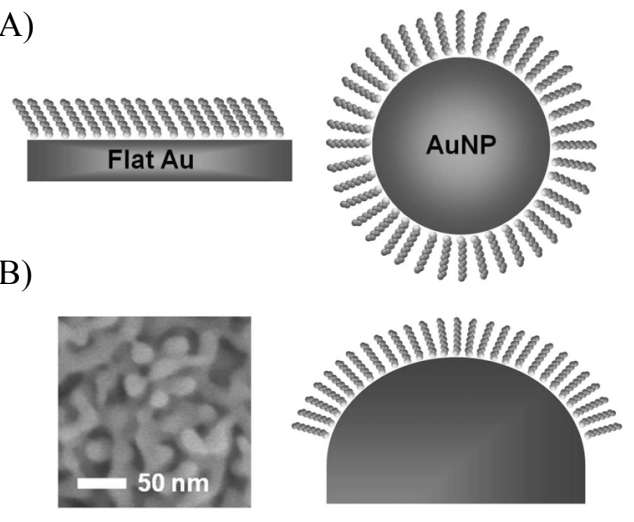

Figure 1. (A) Illustration of alkanethiol monolayers adsorbed on the surface of flat $\mathrm{Au}$ (left) and AuNP (right). (B) Typical scanning microscopic image of a NPG surface (left) and illustration of alkanethiol monolayers adsorbed on the ligament of NPG surfaces.

geneous catalysis and electroanalysis. ${ }^{9-11}$ As shown in Figure 1B, the ligaments on NPG surfaces can be served as curved Au substrates. ${ }^{12}$ The local environment of adsorbed thiolate monolayer on the ligaments of NPG is similar to that on AuNP surfaces. The aim of this work is to examine the characteristic electrochemical behaviors of alkanethiol monolayers adsorbed on the curved Au surfaces compared to those on flat Au surfaces, from which the nature of thiolate monolayers adsorbed on AuNP surfaces can be elucidated.

We first examined the reductive desorption of alkanethiolate monolayers adsorbed on NPG and flat Au surfaces, from which the type and binding characteristics of thiolates at two different Au surfaces can be revealed. ${ }^{13}$ Shown in Figure $2 \mathrm{~A}$ is the cyclic voltammogram for the reductive desorption of dodecanethiol (DT) adsorbed on flat Au surfaces, where a main desorption wave is observed at $-1.06 \mathrm{~V}$. This desorption peak potential is close to that previously reported for DT monolayers on $\mathrm{Au}$ surfaces. ${ }^{8,13,14}$ The origin of a satellite desorption wave observed at $-0.92 \mathrm{~V}$ can be ascribed to the difference in the microscopic roughness on the Au substrates used in this study. ${ }^{15}$ The total charge associated with the reductive desorption of DT on flat Au surfaces is estimated to be $c a .15 \mu \mathrm{C}$. This corresponds to the charge density of $110 \mu \mathrm{C} \mathrm{cm}^{-2}$, which is slightly larger than that observed for alkanethiol SAMs with a monolayer coverage of $(\sqrt{3} \times \sqrt{3}) \mathrm{R} 30^{\circ}$ structures on $\mathrm{Au}(111)$ surfaces. ${ }^{14}$ On NPG surfaces, the reductive peak potential of DT monolayers nega- 
(a)

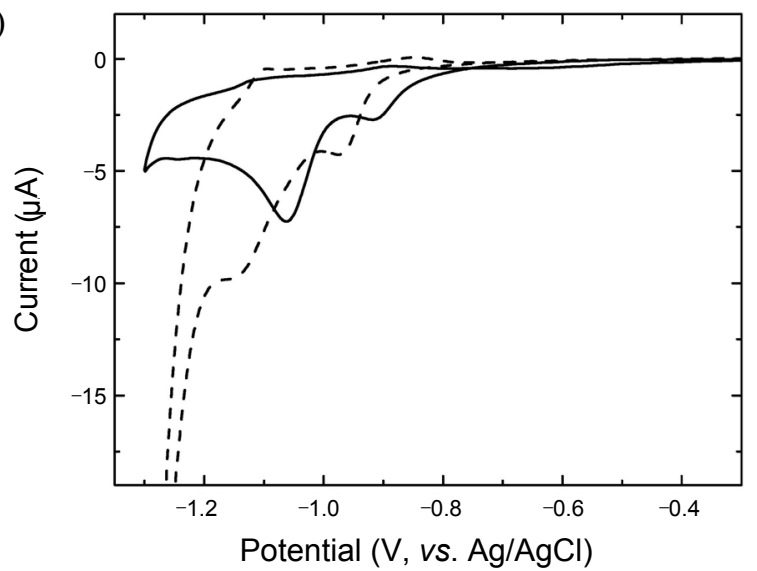

(b)

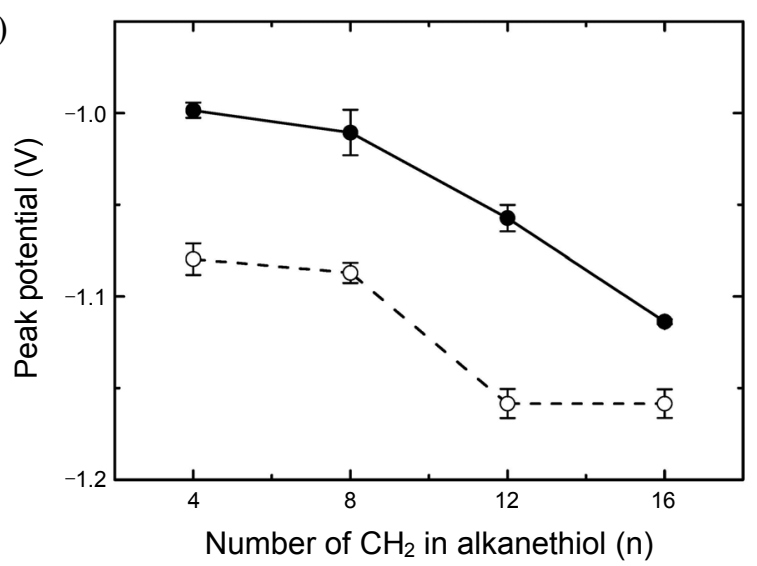

Figure 2. (A) Cyclic voltammograms for the reductive desorption of dodecanethiols adsorbed on flat Au (solid line) and NPG (dashed line) surfaces in $0.1 \mathrm{M} \mathrm{KOH}$. Scan rate: $50 \mathrm{mV} / \mathrm{s}$. (B) Variation of the reductive desorption peak potential of alkanethiols with different length of alkyl chain adsorbed on flat Au (solid line) and NPG (dashed line) surfaces.

tively shifts compared to that on flat Au surfaces, thus the main desorption peak is observed at $-1.16 \mathrm{~V}$. Interestingly, the total charge associated with the reductive desorption of DT on NPG surfaces is measured to be $c a .14 \mu \mathrm{C}$, which is almost same as that found on flat Au surfaces. Because the electrochemical surface area (ESA) of NPG surfaces is 8 times larger than that of flat Au surfaces, the measured charge density associated with the reductive desorption of DT on NPG surfaces is apparently smaller than that expected for the monolayer coverage of DT on NPG. Albeit the reason for this small desorption charge density is not clear, we assume either that all of the NPG surfaces are not covered by DT layer or that only parts of DT layer are desorbing from the surface by the negative potential excursion.

Figure 2B compares the effects of the chain length of alkanethiols on the peak potential of the reductive desorption of alkanethiol monolayers on NPG and flat Au surfaces. The reductive desorption of alkanethiols on NPG surfaces always occurs in more negative potential regions than that on flat $\mathrm{Au}$ surfaces regardless of the chain length of the alkanethiols. On both flat Au and NPG surfaces, the peak potentials of reductive desorption shift in the negative direction as the chain length increases. It was reported that highly roughened nanostructured Au sur-

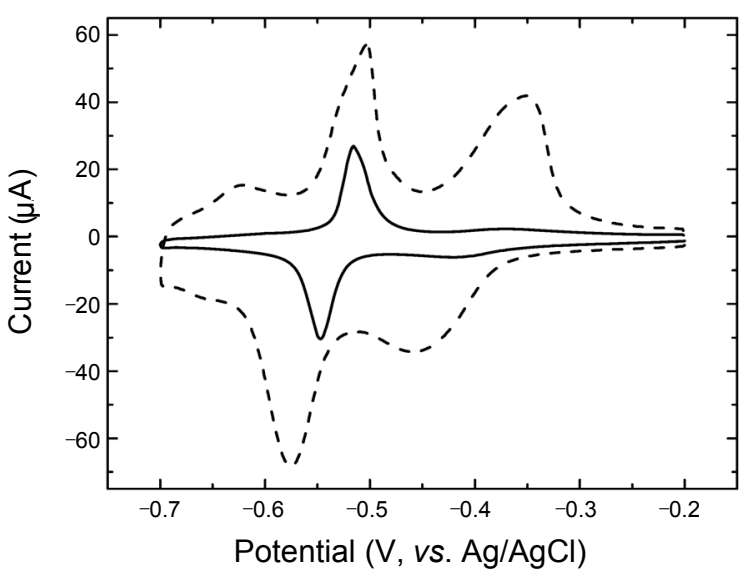

Figure 3. $\mathrm{Pb}$ UPD voltammetric profile of flat $\mathrm{Au}$ (solid line) and $\mathrm{NPG}$ (dashed line) surfaces in $0.1 \mathrm{M} \mathrm{NaOH}+10^{-3} \mathrm{M} \mathrm{Pb}\left(\mathrm{NO}_{3}\right)_{2}$. Scan rate: $50 \mathrm{mV} / \mathrm{s}$.

faces exhibit increased electrochemical stability, where the peak potentials of the reductive desorption of alkanethiol layers are ca. $0.15 \sim 0.2 \mathrm{~V}$ more negative than those on flat Au surfaces. ${ }^{16}$ The values for negative potential shifts on nanostructured $\mathrm{Au}$ are larger than those on NPG (less than $0.1 \mathrm{~V}$ ), and the reason was ascribed to the presence of surface defects such as adatoms and steps on nanostructured Au surfaces.

There are several aspects to be considered to understand the desorption behaviors of alkanethiol monolayers on NPG surfaces. The peak potential for reductive desorption reflects the overall stability of alkanethiol monolayers on Au surfaces governed by the S-Au interaction and the hydrophobic interaction between adjacent alkyl chains. ${ }^{17}$ The negative shift of desorption peak potentials with the increase of chain length of alkanethiols on flat $\mathrm{Au}$ surfaces can be attributed to the increased stability of monolayers due to the greater hydrophobic interactions of longer alkyl chains. ${ }^{8}$ A similar principle can be applied to explain the negative shift of desorption peak potentials with the increase of chain length on NPG surfaces. If we consider the stabilization of the alkanethiol monolayers only based on the hydrophobic interaction between the alkyl chains, the reductive desorption should occur in more positive regions on NPG surfaces than on flat Au surfaces since the self-organization is limited on curved NPG surfaces. However, the cyclic voltammetric results shown in Figure 2 reveal that the reductive desorption of alkanethiol on NPG requires a more negative electrode potential than on bare Au surfaces. This implies that the $\mathrm{S}$-Au interaction becomes stronger on NPG surfaces compared to that on flat Au surfaces, and the additional stability originating from the stronger S-Au interaction is enough to compensate the loss of stability due to the unfavorable self-organization.

It is known that the $\mathrm{S}-\mathrm{Au}$ interaction is stronger at the $\mathrm{Au}(110)$ than the $\mathrm{Au}(111)$ crystalline surfaces, which results in a more negative peak potential for reductive desorption of alkanethiol monolayers at $\mathrm{Au}(110)$ surfaces. ${ }^{13}$ In a previous report, we showed that the NPG surfaces contain a higher amount of $\mathrm{Au}(110)$ domains than flat Au surfaces. ${ }^{11}$ We further examined the surface orientation of NPG surfaces by an underpotential deposition of lead (Pb UPD). The anodic stripping peak po- 
(a)

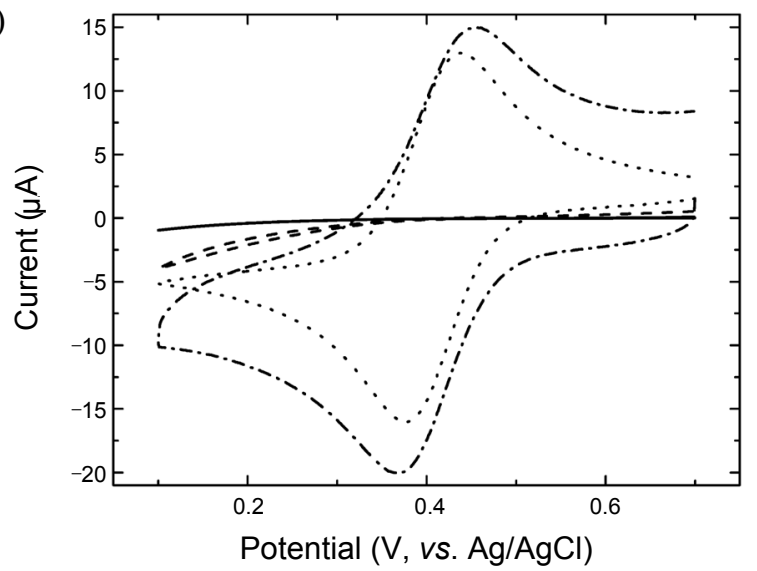

(b)

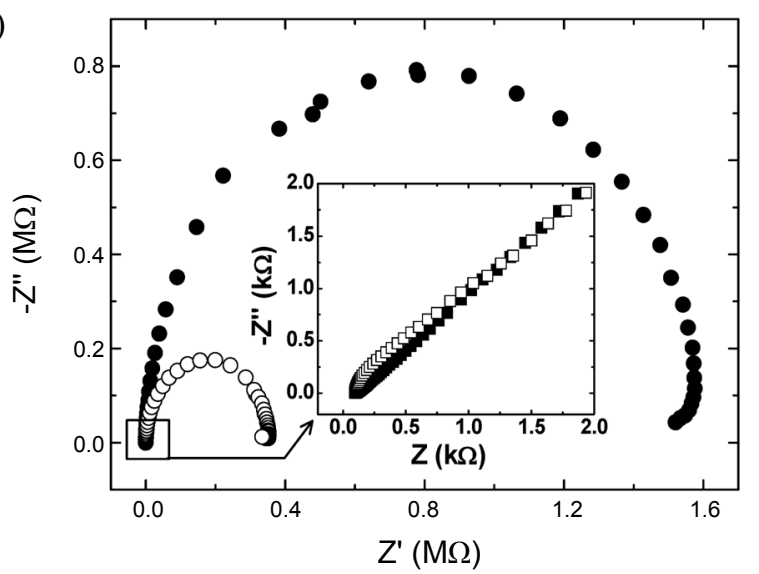

Figure 4. (A) Cyclic voltammograms obtained in $1 \mathrm{mM} \mathrm{Fe}(\mathrm{CN})_{6}{ }^{3-}+$ $0.1 \mathrm{M} \mathrm{H}_{2} \mathrm{SO}_{4}$ on dodecanethiol-adsorbed flat $\mathrm{Au}$ (solid line) and NPG (dashed line) surfaces. Dotted line and dash-dotted line were obtained on unmodified flat Au and NPG surfaces, respectively. Scan rate: 50 $\mathrm{mV} / \mathrm{s}$. (B) Nyquist plots of dodecanethiol-adsorbed flat Au (solid circle) and NPG (empty circle) surfaces obtained from EIS measurements in $1 \mathrm{mM} \mathrm{Fe}(\mathrm{CN})_{6}{ }^{3-}+0.1 \mathrm{M} \mathrm{H}_{2} \mathrm{SO}_{4}$. Inset was obtained on unmodified flat $\mathrm{Au}$ (filled square) and NPG (empty square) surfaces.

tentials in UPD can be used as indicators to characterize the structure of electrode surfaces. ${ }^{18}$ In Figure 3, an anodic peak at $-0.35 \mathrm{~V}$ is clearly visible from the UPD curves obtained on the NPG surfaces, which is not observed on a flat Au surface. This peak can be assigned to the anodic stripping of $\mathrm{Pb}$ on $\mathrm{Au}(110)$ facets of the NPG surfaces. The anodic peaks at around $-0.51 \mathrm{~V}$, observed on both of the flat Au and NPG surfaces, correspond to the (111) facet on Au surfaces. This result strongly suggests the presence of $\mathrm{Au}(110)$ facets on NPG surfaces. Therefore, the more negative desorption potential of alkanethiols on NPG than flat Au surfaces can be ascribed to the enhanced stability of monolayers due to the stronger S-Au interaction on NPG despite of the reduced self-organization effect on the curved surface structures.

Electron transfer behaviors of redox active species through the alkanethiol monolayers can serve as indicators to examine the intrinsic properties of the adsorbed monolayers on Au surfaces. ${ }^{19,20}$ We investigated the electron transfer behaviors of $\mathrm{Fe}(\mathrm{CN})_{6}{ }^{3-}$ species through alkanethiol monolayers adsorbed on flat Au and NPG surfaces. As shown in Figure 4A, a well-de- fined redox wave observed on a unmodified flat Au surfaces (dotted line), while there is no discernable faradaic current on the DT-adsorbed flat Au surface (solid line). This indicates that the electron transfer between the flat Au surface and $\mathrm{Fe}(\mathrm{CN})_{6}{ }^{3-}$ species is greatly attenuated by the monolayer of DT. When the DT monolayers adsorb on NPG surfaces (dashed line), a slight increase in the current is observed, which implies that the electron transfer through DT monolayers on NPG surfaces is somewhat recovered compared to that on flat Au surfaces. Figure 4A also shows that the cyclic voltammogram obtained on unmodified NPG surfaces (dash-dotted line), where the electron transfer between the NPG surface and $\mathrm{Fe}(\mathrm{CN})_{6}{ }^{3-}$ species is slightly attenuated compared to the flat Au surfaces. Note that the current level on NPG surfaces is virtually same as that on flat Au surfaces after double-layer correction, which implies that the effective surface area utilized for the redox process of $\mathrm{Fe}(\mathrm{CN})_{6}{ }^{3-}$ species is almost identical on both of the NPG and flat $\mathrm{Au}$ surfaces.

The charge-transfer resistances of the $\mathrm{Fe}(\mathrm{CN})_{6}{ }^{3-}$ redox reaction on the DT monolayers on flat Au and NPG surfaces were measured by the Nyquist plots from electrochemical impedance spectroscopy (EIS) measurements (Figure 4B). The redox processes for $\mathrm{Fe}(\mathrm{CN})_{6}{ }^{3-}$ species on unmodified NPG and flat $\mathrm{Au}$ surfaces are mass transport limited over the whole frequency range, and these fast electron transfer kinetics are consistent with the cyclic voltammetric observations. The charge-transfer resistance of the redox reaction involving the $\mathrm{Fe}(\mathrm{CN})_{6}{ }^{3-}$ species on the DT monolayers on flat Au surfaces is estimated to be $1.5 \times 10^{6} \Omega$, which is similar to the previously reported value. ${ }^{21}$ The charge-transfer resistance on the DT monolayers on NPG surfaces is measured to be $3.4 \times 10^{5} \Omega$, which is an order of magnitude smaller than that found on flat Au surfaces.

The decrease in the charge-transfer resistance clearly indicates that the electron transfer is more facile on the DT-monolayers adsorbed on NPG than on flat Au surfaces. Porter et al. suggested that the electron transfer process through alkanethiol monolayers on Au surfaces can occur in two ways. ${ }^{4}$ One way is the electron transfer through the monolayer films via a tunneling process, and the other way is via permeating of redox species through the monolayer and reacting at the electrode surface. The monolayers of DT on flat Au surfaces are self-assembled, thus the electron transfer occurs via a tunneling process. In this case, the charge-transfer resistance would increase as the length of the alkyl chain in alkanethiols increases. The solid line in Figure 5 shows that the charge-transfer resistance of $\mathrm{Fe}(\mathrm{CN})_{6}{ }^{3-}$ species at alkanethiol monolayers adsorbed on flat Au surfaces increases as the chain length increases, and a similar trend has been previously reported. ${ }^{22}$ On the other hand, the self-organization of the monolayers of alkanethiols on curved NPG surfaces is limited; therefore, there are more chances for redox species permeating through the monolayers to react at the Au surfaces. The enhanced electron transfer kinetics at DT monolayer on NPG surfaces can be partly explained by this mechanism. The dashed line in Figure 5 revels that the chargetransfer resistances at alkanethiol monolayers adsorbed on NPG surfaces are relatively invariant regardless of the chain length of alkanethiols. We assume that the permeation of the redox species through the monolayers of alkanethiols is not much 


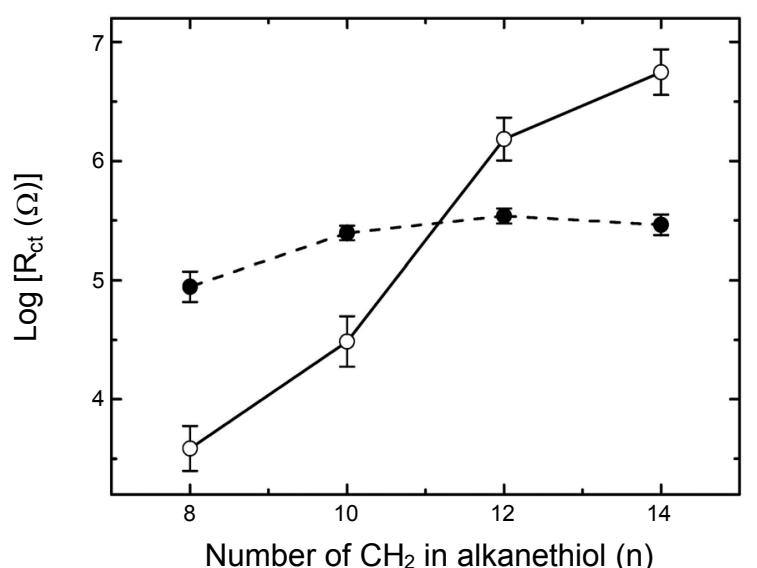

Figure 5. Variation of the charge-transfer resistance estimated from the Nyquist plots of dodecanethiol-adsorbed flat $\mathrm{Au}$ (solid) and NPG (dashed) surfaces by EIS measurements in $1 \mathrm{mM} \mathrm{Fe}(\mathrm{CN})_{6}{ }^{3-}+0.1 \mathrm{M}$ $\mathrm{H}_{2} \mathrm{SO}_{4}$.

affected by the length of the alkyl chains.

In summary, we investigated the electrochemical properties of alkanethiol monolayers adsorbed on NPG surfaces by cyclic voltammetry and electrochemical impedance spectroscopy, and the results are compared to those on flat Au surfaces. The reductive desorption of alkanethiols on NPG surfaces is observed in more negative potential regions than that on flat Au surfaces due the stronger S-Au interaction on NPG surfaces. While the electron transfer through alkanethiol monolayers on flat $\mathrm{Au}$ surfaces occurs via a tunneling process through the monolayer films, the redox species can permeate through the monolayers on NPG surfaces to transfer the electrons to the Au surfaces. The results presented here will help to elucidate the intrinsic electrochemical properties of alkanethiol monolayers adsorbed on curved Au surfaces, particularly on the surface of AuNPs.

\section{Experimental Section}

NPG electrodes were prepared by electrodeposition of Ag-Au alloy layers followed by selective dissolution of $\mathrm{Ag}$ in concentrated nitric acid. $\mathrm{Au}$ films evaporated onto silicon $(\mathrm{Au} / \mathrm{Si})$ wafers (KMAC, Korea) were employed as Au substrates, on which the $\mathrm{Ag}-\mathrm{Au}$ alloy layers were electrodeposited from a solution containing $27 \mathrm{mM} \mathrm{KAg}(\mathrm{CN})_{2}, 15 \mathrm{mM} \mathrm{KAu}(\mathrm{CN})_{2}$, and $0.25 \mathrm{M}$ $\mathrm{Na}_{2} \mathrm{CO}_{3}$ at $-0.95 \mathrm{~V}$ with a total deposition charge of $0.01 \mathrm{C}$ The Ag-Au alloy layers on $\mathrm{Au} / \mathrm{Si}$ wafers were transferred to a concentrated nitric acid to dissolve Ag. The prepared NPG electrode was confined in a Viton O-ring with an inner diameter of $2.9 \mathrm{~mm}$ and used as a working electrode. Electrochemical measurements were conducted using a CHI 660 (CH Instrument) potentiostat. $\mathrm{Pt}$ wire and $\mathrm{Ag} / \mathrm{AgCl}$ electrodes were used as counter and reference electrodes, respectively. All potentials are reported relative to the $\mathrm{Ag} / \mathrm{AgCl}$ reference electrode (3 M $\mathrm{KCl}$ ). Modification of the Au surfaces with the monolayers of alkanethiols was performed by dipping Au surfaces in $1 \mathrm{mM}$ alkanethiol solutions in ethanol for 24 hours. Scanning electron microscopy (SEM) characterization was performed using a LEO 1530 Field Emission SEM (Carl Zeiss) with an acceleration voltage of $5 \mathrm{kV}$.

Acknowledgments. This research was financially supported by the Ministry of Education, Science Technology (MEST) and Korea Institute for Advancement of Technology (KIAT) through the Human Resource Training Project for Regional Innovation. This work was supported by the grant of the Korean Ministry of Education, Science and Technology (The Regional Core Research Program/Chungbuk BIT Research-Oriented University Consortium).

\section{References}

1. Ulman, A. Chem. Rev. 1996, 96, 1533.

2. Love, J. C.; Estroff, L. A.; Kriebel, J. K.; Nuzzo, R. G.; Whitesides, G. M. Chem. Rev. 2005, 105, 1103 .

3. Strong, L.; Whitesides, G. M. Langmuir 1988, 4, 546.

4. Porter, M. D.; Bright, T. B.; Allara, D. L.; Chidsey, C. E. D. J. Am. Chem. Soc. 1987, 109, 3559.

5. Camillone, N.; Chidsey, C. E. D.; Liu, G.; Scoles, G. J. Chem. Phys. 1993, 98, 4234.

6. Dubois, L. H.; Zegarski, B. R.; Nuzzo, R. G. J. Chem. Phys. 1993, 98,678

7. Walczak, M. M.; Alves, C. A.; Lamp, B. D.; Porter, M. D. J. Electroanal. Chem. 1995, 396, 103.

8. Widrig, C. A.; Chung, C.; Porter, M. D. J. Electroanal. Chem. $1991,310,335$.

9. Wittstock, A.; Zielasek, V.; Biener, J.; Friend, C. M.; Baumer, M. Science 2010, 327, 319.

10. Liu, Z.; Du, J.; Qiu, C.; Huang, L.; Ma, H.; Shen, D.; Ding, Y. Electrochem. Commun. 2009, 11, 1365.

11. Seo, B.; Kim, J. Electroanalysis 2010, 22, 939.

12. Erlebacher, J. J. Electrochem. Soc. 2004, 151, C614.

13. Zhong, C. J.; Zak, J.; Porter, M. D. J. Electroanal. Chem. 1997, $421,9$.

14. Qu, D.; Kim, B.-C.; Lee, C.-W. J.; Uosaki, K. Bull. Korean Chem. Soc. 2009, 30, 2549.

15. Wong, S. S.; Porter, M. D. J. Electroanal. Chem. 2000, 485, 135.

16. Cortes, E.; Rubert, A. A.; Benitez, G.; Carro, P.; Vela, M. E.; Salvarezza, R. C. Langmuir 2009, 25, 5661.

17. Mirsky, V. M. Trac-Trends Anal. Chem. 2002, 21, 439

18. Hernandez, J.; Solla-Gullon, J.; Herrero, E. J. Electroanal. Chem. 2004, 574, 185.

19. Protsailo, L. V.; Fawcett, W. R. Electrochim. Acta 2000, 45, 3497.

20. Campina, J. M.; Martins, A.; Silva, F. J. Phys. Chem. C 2009, 113, 2405.

21. Xing, Y. F.; O'Shea, S. J.; Li, S. F. Y. J. Electroanal. Chem. 2003, $542,7$.

22. Bradbury, C. R.; Zhao, J. J.; Fermin, D. J. J. Phys. Chem. C 2008, $112,10153$. 